\title{
STABILITY OF PERIODIC SOLUTIONS OF THE NONLINEAR STRING*
}

\author{
BY R. W. DICKEY (University of Wisconsin, Madison)
}

1. Introduction. In 1945 Carrier [1] proposed an approximate nonlinear theory to describe the vibration of a string. Narasimha [2] has shown that this approximate theory may be obtained from the exact string equations in a systematic way.

Although Carrier's equation is simpler than the exact equations, there remain unresolved questions about its solution. Indeed, there remain questions about the appropriate conditions on the initial data to guarantee global existence of a smooth solution [3-6]. Even so, it is of interest to obtain information about the qualitative behavior of solutions, and in fact there has been considerable research on the behavior of solutions to Carrier's equation and related equations when viscous damping is present and when periodic forcing is applied [2,7-12].

An interesting feature of the Carrier equation is that, even though it is nonlinear, it has an infinite set of periodic solutions (modes) similar to the modes of the linear wave equation (cf. [3]). However, since the Carrier equation is nonlinear it is not possible to build up more complicated solutions by linear superposition of these special solutions.

It is the aim of this paper to study the stability of the modal vibrations of the Carrier equation. In particular, it will be shown that an arbitrary small perturbation of data corresponding to a pure modal vibration has only a small effect. A similar result holds for the linear wave equation and is in agreement with experiment.

2. Preliminaries. For simplicity it will be assumed that the string has length $\pi$. In this case the Carrier equation can be written

$$
w_{t t}-\left(\lambda^{2}+\frac{2}{\pi} \int_{0}^{\pi} w_{x}(x, t)^{2} d x\right) w_{x x}=0
$$

where $w(x, t)$ and $\lambda^{2}$ are essentially the transverse displacement of the string and $\lambda^{2}$ is the equilibrium tension in the string (cf. $[1,2])$. It may be noted that linearization of $(2.1)$ leads to the ordinary linear wave equation.

If the ends of the string are held fixed and the initial position and velocity are prescribed it follows that solutions of (2.1) must satisfy the conditions

$$
\begin{gathered}
w(0, t)=w(\pi, t)=0 \\
w(x, 0)=f(x), \quad w_{t}(x, 0)=g(x),
\end{gathered}
$$

\footnotetext{
* Received September 11, 1979. This research was supported by the National Science Foundation, Grant No. MPS 75-06366.
} 
where $f(x)$ and $g(x)$ are known functions. In this paper it will be assumed that the initial data (2.3) has the form of a finite Fourier series, i.e.

$$
f(x)=\sum_{j=1}^{N} f_{j} \sin j x, \quad g(x)=\sum_{j=1}^{N} g_{j} \sin j x ;
$$

such data is said to be "quiescent" (cf. [13]).

It is easily verified that the solution of (2.1) satisfying the above conditions has the form

$$
w(x, t)=\sum_{j=1}^{N} T_{j}(t) \sin j x .
$$

if the functions $T_{j}(t)$ satisfy the system of nonlinear ordinary differential equations $(\dot{T}=d / d t)$

$$
\ddot{T}_{j}+j^{2}\left(\lambda^{2}+\sum_{l=1}^{N} l^{2} T_{l}^{2}\right) T_{j}=0, \quad j=1,2, \ldots, N,
$$

and the initial conditions

$$
T_{j}(0)=f_{j}, \quad \dot{T}_{j}(0)=g_{j} .
$$

The system (2.6) is a coupled nonlinear system.

In the special case that $f_{j}=g_{j}=0$ for $j \neq k$ the system (2.6) reduces to a single nonlinear equation

$$
\ddot{T}_{k}+k^{2}\left(\lambda^{2}+k^{2} T_{k}^{2}\right) T_{k}=0 .
$$

It is well known that every solution of (2.8) is periodic (cf. [14]). Thus the Carrier equation has special periodic solutions of the form

$$
w(x, t)=T_{k}(t) \sin k x .
$$

These periodic solutions are the modes referred to in Sec. 1. In general, however, solutions of the system (2.6) are not periodic.

3. Stability. It is convenient to introduce the notation

$$
\varepsilon=\left(f_{k}^{2}+g_{k}^{2}\right)^{1 / 2}, \quad \delta=\left(\sum_{\substack{l=1 \\ l \neq k}}^{N}\left(f_{l}^{2}+g_{l}^{2}\right)\right)^{1 / 2}
$$

We assume that both $\varepsilon$ and $\delta$ are small quantities, and in addition

$$
\mu=\delta / \varepsilon
$$

is small. Thus we treat the case of small initial data in which the data for one mode dominates the rest.

We have observed in Sec. 2 that if $\delta=0$ the solution of (2.6) is periodic. If $\delta \neq 0$ then in general the solution of (2.6) is not periodic. However, since the periodic modal vibration of the string may be observed, it would suggest that when $\mu$ is sufficiently small the solution lies close to a periodic vibration. If this is the case these periodic solutions are said to be stable. 
In order to decide on the stability or instability of the modal vibrations of the string it is convenient to introduce the change of variable

$$
\begin{aligned}
& T_{j}=\delta V_{j} \quad j \neq k, \\
& T_{k}=\varepsilon V_{k} .
\end{aligned}
$$

In terms of these new variables the system (2.6) may be written

$$
\ddot{V}_{j}+j^{2}\left(\lambda^{2}+\varepsilon^{2}\left(k^{2} V_{k}^{2}+\mu^{2} \sum_{\substack{l=1 \\ l \neq k}}^{N} l^{2} V_{l}^{2}\right)\right) V_{j}=0, \quad j=1, \ldots, N .
$$

The solution of (3.4) must satisfy the conditions

$$
\begin{array}{lll}
V_{j}(0)=A_{j}=f_{j} / \delta, & \dot{V}_{j}(0)=B_{j}=g_{j} / \delta, & j \neq k \\
V_{k}(0)=A_{k}=f_{k} / \varepsilon, & \dot{V}_{k}(0)=B_{k}=g_{k} / \varepsilon . &
\end{array}
$$

It is a consequence of the definition of $\varepsilon$ and $\delta$ that $0 \leq\left|A_{n}\right|,\left|B_{n}\right| \leq 1(n=1, \ldots, N)$.

The solution of (3.4) depends on the two parameters $\varepsilon$ and $\mu$. We look for solutions in the form of a perturbation expansion

$$
V_{j}(t ; \varepsilon, \mu)=\sum_{l=0}^{\infty} V_{j}^{(l)}(t ; \varepsilon) \mu^{l} .
$$

Combining (3.4), (3.5), and (3.6), we find that $V_{j}^{(0)}$ must satisfy

$$
\begin{gathered}
\ddot{V}_{j}^{(0)}+j^{2}\left(\lambda^{2}+\varepsilon^{2} k^{2} V_{k}^{(0) 2}\right) V_{j}^{(0)}=0, \quad j=1,2, \ldots, N \\
V_{j}^{(0)}(0)=A_{j}, \quad \dot{V}_{j}^{(0)}(0)=B_{j} .
\end{gathered}
$$

The object is to study the solution of the system (3.7). This system is peculiar in that the equation with $j=k$, i.e.

$$
\ddot{V}_{k}^{(0)}+k^{2}\left(\lambda^{2}+\varepsilon^{2} k^{2} V_{k}^{(0)}\right) V_{k}^{(0)}=0
$$

is nonlinear while the remaining equations with $j \neq k$ are linear with coefficients determined by the solutions of (3.9). Every solution of (3.9) is periodic. Thus the system (3.7) with $j \neq k$ is a linear system of equations with periodic coefficients, i.e. Hill's type equations. It is well known that the solutions of such equations may be bounded or unbounded depending on various features of the equation (cf. [15]).

The actual solution of the Carrier equation can be written in the form (cf. (2.5))

$$
w(x, t)=\varepsilon\left(V_{k}^{(0)}(t) \sin k x+\mu \sum_{\substack{l=1 \\ l \neq k}}^{N} V_{l}^{(0)}(t) \sin l x\right)+\cdots .
$$

The function $V_{k}^{(0)}$ is periodic. If the functions $V_{l}^{(0)}$ are bounded for all time the solution (3.10) would be indistinguishable from a pure modal vibration when $\mu$ is sufficiently small. Thus the object is to show that the functions $V_{l}^{(0)}$ are uniformly bounded.

Since the solution of (3.9) is periodic it is convenient to introduce a change of variable

$$
t=\alpha(\varepsilon) \tau
$$


so that $V_{k}^{(0)}$ is periodic with smallest period $2 \pi$ in $\tau$. In addition we define

$$
S_{j}=\alpha V_{j}^{(0)}, \quad j=1,2, \ldots, N .
$$

In these new variables (3.7) and (3.8) become $\left({ }^{\prime}=d / d \tau\right)$

$$
\begin{gathered}
S_{j}^{\prime \prime}+j^{2}\left(\lambda^{2} \alpha^{2}+\varepsilon^{2} k^{2} S_{k}^{2}\right) S_{j}=0, \quad j=1,2, \ldots, N \\
S_{j}(0)=\alpha A_{j}, \quad S_{j}^{\prime}(0)=\alpha^{2} B_{j} .
\end{gathered}
$$

The function $\alpha(\varepsilon)$ is determined so that the minimum period of the solution of

$$
\begin{aligned}
& S_{k}^{\prime \prime}+k^{2}\left(\lambda^{2} \alpha^{2}+\varepsilon^{2} k^{2} S_{k}^{2}\right) S_{k}=0 \\
& S_{k}(0)=\alpha A_{k}, \quad S_{k}^{\prime}(0)=\alpha^{2} B_{k}
\end{aligned}
$$

has the value $2 \pi$. Actually $\alpha(\varepsilon)$ and $S_{k}(\tau ; \varepsilon)$ can be determined explicitly by assuming expansions of the form

$$
\begin{gathered}
S_{k}(\tau ; \varepsilon)=\sum_{l=0}^{\infty} S_{k}^{(l)}(\tau) \varepsilon^{l} \\
\alpha(\varepsilon)=\sum_{l=0}^{\infty} \alpha^{(l)} \varepsilon^{l} .
\end{gathered}
$$

In any case it is easily shown that

$$
\begin{gathered}
S_{k}(\tau ; \varepsilon)=\frac{A_{k}}{\lambda k} \cos \tau+\frac{B_{k}}{\lambda^{2} k^{2}} \sin \tau+\cdots \\
\alpha(\varepsilon)=\frac{1}{\lambda k}-\frac{3}{8 \lambda k}\left(\lambda^{2} k^{2} A_{k}^{2}+B_{k}^{2}\right) \varepsilon^{2}+\cdots
\end{gathered}
$$

The function $S_{k}(\tau ; \varepsilon)$ is periodic of period $2 \pi$. The function $S_{k}(\tau ; \varepsilon)^{2}$ is periodic of period $\pi$. Thus the system of equations (3.13) with $j \neq k$ is a linear system with coefficients of period $\pi$. In order to decide whether the solutions of this linear system are bounded or unbounded it is only necessary to construct the stability diagram for each equation. Specifically, we consider the equation

$$
S^{\prime \prime}+\left(h(\varepsilon)+\varepsilon^{2} j^{2} k^{2} S_{k}^{2}\right) S=0
$$

where we note that if $h(\varepsilon)=j^{2} \lambda^{2} \alpha^{2}(\varepsilon)$ Eq. (3.21) is simply the $j$ th equation of the system (3.13). If we look in the $(h, \varepsilon)$ plane, Floquet theory (cf. [16, pp. 78-81, 99]) guarantees that from each of the points $\left(n^{2}, 0\right)$ there bifurcates a pair of curves $h=H_{n}^{(1)}(\varepsilon)$ and $h=H_{n}^{(2)}(\varepsilon)$ (cf. Fig. 3.1). These curves divide the $(h, \varepsilon)$ plane into regions in which every solution is bounded (stable region) and regions in which there is an unbounded solution (unstable region). Thus if one knew the functions $H_{n}^{(1)}(\varepsilon)$ and $H_{n}^{(2)}(\varepsilon)$ it would be possible to decide whether the point $\left(j^{2} \lambda^{2} \alpha^{2}(\varepsilon), \varepsilon\right)$ lies in the stable or unstable regions. In certain cases it is possible to make this decision without actually computing $H_{n}^{(1)}(\varepsilon)$ and $H_{n}^{(2)}(\varepsilon)$. In particular we note that $\lim _{\varepsilon \rightarrow 0} j^{2} \lambda^{2} \alpha^{2}(\varepsilon)=j / k$. If $j / k \neq n$ for any integer $n$ it follows that $j / k$ is an interior point of a region of stability. Therefore we conclude that if $\varepsilon$ is sufficiently small and $j / k \neq n$ the solution is bounded.

This result does not decide the question of stability for all the equations (3.13), since it does not exclude the growth of modes which are integral multiples of $k$. In particular, if 


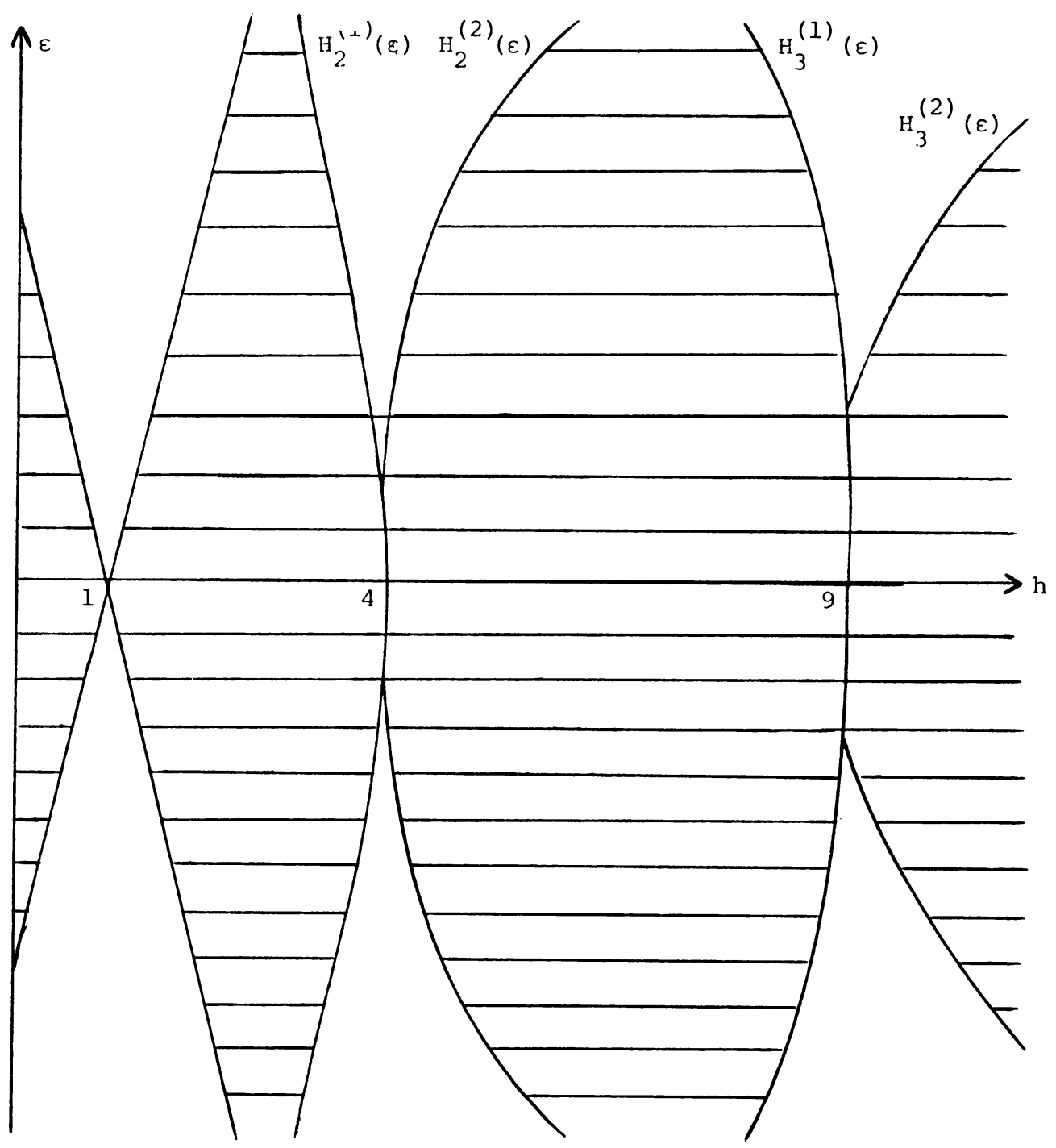

Fig. 3.1

the dominant data had been given in the first mode, i.e. if $k=1$, then $j / k$ is always an integer. In the case $j / k=n$ the point $\left(j^{2} \lambda^{2} \alpha^{2}(0), 0\right)=\left(n^{2}, 0\right)$ is on the boundary of the region of stability and it must be decided whether the curve (cf. (3.20))

$$
j^{2} \lambda^{2} \alpha^{2}(\varepsilon)=n^{2}-\frac{3 n^{2}}{4}\left(\lambda^{2} k^{2} A_{k}^{2}+B_{k}^{2}\right) \varepsilon^{2}+\cdots
$$

enters the stable or unstable region. 
The function $H_{n}^{(1)}(\varepsilon)$ is determined by the requirement that $H_{n}^{(1)}(0)=n^{2}$ and the solution of

$$
\begin{gathered}
S^{\prime \prime}+\left(H_{n}^{(1)}(\varepsilon)+\varepsilon^{2} j^{2} k^{2} S_{k}^{2}\right) S=0 \\
S(0)=1, \quad S^{\prime}(0)=0,
\end{gathered}
$$

where $S_{k}(\tau ; \varepsilon)$ is given by $(3.19)$, should be periodic. Similarly $H_{n}^{(2)}(\varepsilon)$ is determined by the requirement that $H_{n}^{(2)}(0)=n^{2}$ and the solution of

$$
\begin{gathered}
S^{\prime \prime}+\left(H_{n}^{(2)}(\varepsilon)+\varepsilon^{2} j^{2} k^{2} S_{k}^{2}\right) S=0 \\
S(0)=0, \quad S^{\prime}(0)=1
\end{gathered}
$$

should be periodic (cf. [16]). We can exclude the cases $n=0,1$ since $j / k$ cannot take on these values.

The above problems can be solved by perturbation where successive terms in the expansion of $H_{n}^{(1)}(\varepsilon)$ and $H_{n}^{(2)}(\varepsilon)$ are determined by the condition that the solutions be periodic. In any case it may be shown that

$$
\begin{aligned}
& H_{n}^{(1)}(\varepsilon)=n^{2}-\frac{n^{2}}{2}\left(\lambda^{2} k^{2} A_{k}^{2}+B_{k}^{2}\right) \varepsilon^{2}+\cdots \\
& H_{n}^{(2)}(\varepsilon)=n^{2}-\frac{n^{2}}{2}\left(\lambda^{2} k^{2} A_{k}^{2}+B_{k}^{2}\right) \varepsilon^{2}+\cdots
\end{aligned}
$$

Thus $H_{n}^{(1)}(\varepsilon)$ and $H_{n}^{(2)}(\varepsilon)$ agree up to terms of second order. Comparing (3.22) with (3.27) and (3.28) we conclude that the curve $\left(j^{2} \lambda^{2} \alpha^{2}(\varepsilon), \varepsilon\right)$ enters the stable region. Therefore even in the case $j / k=n$ the solution of (3.13) is bounded when $\varepsilon$ is sufficiently small. The linear stability of the single mode periodic vibrations (with small amplitude) is a consequence.

\section{REFERENCES}

[1] G. F. Carrier, On the nonlinear vibration problem of the elastic string, Quart. Appl. Math. 3, 157 (1945)

[2] R. Narasimha, Non-linear vibration of an elastic string, J. Sound Vib. 8, 134 (1968)

[3] R. W. Dickey, Infinite systems of nonlinear oscillation equations related to the string, Proc. Amer. Math. Soc. 23, 459 (1969)

[4] T. Nishida, A note on the nonlinear vibrations of an elastic string, Mem. Fac. Eng., Kyoto Univ., 34 (1971)

[5] R. W. Dickey, The initial value problem for a nonlinear semi-infinite string, Proc. Roy. Soc. Edin. 82A, 19 (1978)

[6] S. C. Hu, The initial value problem for a non-linear infinite string, Ph.D. Thesis, State University of New York at Buffalo, 1979

[7] G. S. S. Murthy and B. S. Ramakrishna, Non-linear character of resonance in stretched strings, J. Acoust. Soc. Amer. 38, 461 (1965)

[8] J. W. Miles, Stability of forced oscillations of a vibrating string, J. Acoust. Soc. Amer. 38, 1855 (1965)

[9] G. V. Anand, Non-linear resonance in stretched strings with viscous damping, J. Acoust. Soc. Amer. 40, 1517 (1966)

[10] E. W. Lee, Non-linear forced vibration of a stretched string, Br. J. Appl. Phys. 8, 411 (1957)

[11] D. W. Oplinger, Frequency response of a non-linear stretched string, J. Acoust. Soc. Amer. 32, 1529 (1960)

[12] R. W. Dickey, Infinite systems of nonlinear oscillation equations with linear damping, SIAM J. Appl. Math. 19, 208 (1970) 
[13] E. L. Reiss and B. J. Matkowsky, Nonlinear dynamic buckling of a compressed elastic column, Quart. Appl. Math. 29, 245 (1971)

[14] J. J. Stoker, Nonlinear vibrations, Interscience, New York, 1950

[15] L. Brillouin, Wave propagation in periodic structures, Dover, New York, 1946

[16] E. A. Coddington and N. Levinson, Theory of ordinary differential equations, McGraw-Hill, New York, 1955 